\title{
Is it really post-irradiation morphea or oleoma of the breast? -A case report and literature review
}

\author{
Han Shin Lee ${ }^{1}$, Eun Jung Jung ${ }^{1}$, Jae Myung Kim², Ju Yeon Kim², In Kyeong Kim ${ }^{1}$, Jae Ri Kim ${ }^{1}$, \\ Tae Han Kim ${ }^{1}$, Jae Yool Jang ${ }^{1}$, Jung Woo Woo ${ }^{1}$, JinKwon Lee ${ }^{1}$, Taejin Park ${ }^{1}$, Sang Ho Jeong ${ }^{1}$, Eun Cho ${ }^{3}$, \\ Hyo Jung $\mathrm{An}^{4}$
}

${ }^{1}$ Department of Surgery, Gyeongsang National University Changwon Hospital, Gyeongsang National University College of Medicine, Changwon, Korea; ${ }^{2}$ Department of Surgery, Gyeongsang National University Hospital, Gyeongsang National University College of Medicine, Jinju, Korea; ${ }^{3}$ Department of Radiology, Gyeongsang National University Changwon Hospital, Gyeongsang National University College of Medicine, Changwon, Korea; ${ }^{4}$ Department of Pathology, Gyeongsang National University Changwon Hospital, Gyeongsang National University College of Medicine, Changwon, Korea

Correspondence to: Eun Jung Jung, MD, PhD. Department of Surgery, Gyeongsang National University Changwon Hospital, Gyeongsang National University College of Medicine, 11, Samjeongja-ro, Seongsan-Gu, Changwon 51472, Korea. Email: drjej@gnu.ac.kr.

\begin{abstract}
We report the case of a 65 -year-old woman who underwent breast-conserving surgery (BCS) and radiotherapy for breast cancer with hyperpigmentation and skin thickening of the treated breast site 10 years after the surgery. The patient was injected with a liquid foreign body in both breasts 30 years ago. Theses clinical features were considered scleroderma, post-irradiation morphea (PIM), and recurrent breast cancer for differential diagnosis. We performed breast magnetic resonance imaging (MRI), however, the patient had no abnormal findings. Owing to the pain, increased hyperpigmentation, and possibility of cancer recurrence, the patient underwent a simple mastectomy. The final pathologic diagnosis was oleoma with post-radiation fibrosis among drug-induced and toxic scleroderma-like disorders. The patient tolerated surgical therapy without complications. This case report highlight is that difficult to distinguish between PIM and oleoma in patients with a complex history. In this case, the patient had both a history of radiotherapy and a history of foreign body injection, making the clinical diagnosis difficult. PIM and oleoma are non-malignant but can impair a patient's quality of life owing to symptoms and the clinical presentation is similar to that of local recurrence of breast cancer. Thus, arriving at the correct diagnosis typically requires a multidisciplinary approach, including imaging follow-up, skin punch biopsy, or surgery for a definitive diagnosis.
\end{abstract}

Keywords: Breast; radiotherapy; oleoma; morphea; case report

Submitted Aug 12, 2021. Accepted for publication Nov 03, 2021.

doi: $10.21037 /$ gs-21-549

View this article at: https://dx.doi.org/10.21037/gs-21-549

\section{Introduction}

After breast-conserving surgery (BCS) for breast cancer, radiotherapy is the standard method, as it improves overall survival and is associated with a decreased risk of breast cancer death and recurrence (1). However, radiationinduced dermatitis or skin changes are very common in patients with breast cancer $(2,3)$. Local irritation and drying of the skin is observed in the first 3 months of radiation exposure, while late adverse effects, including sclerodermatous changes, skin telangiectasia, atrophy, skin necrosis, and secondary malignancies, may occur from months to years after radiotherapy (4). Post-irradiation morphea (PIM) is a very rare late adverse effect of breast irradiation, characterized by the deposition of thickened

\footnotetext{
$\wedge$ ORCID: 0000-0001-8928-0624.
} 
collagen and abnormal fibroblast activity (5).

For breast augmentation, subcutaneous injection of liquid foreign material, such as paraffin or vegetable oil has been used for more than a century (6). Liquid foreign body induced paraffinoma, siliconoma, or oleoma may occur per the injected substance.

Herein, we present a rare case of oleoma with combined post-radiation fibrosis, necessary for differential diagnosis of PIM.

We present the following case in accordance with the CARE reporting checklist (available at https://dx.doi. org/10.21037/gs-21-549).

\section{Case presentation}

\section{Clinical bistory}

Five years ago, a 65-year-old woman with a foreign body injection at both breasts approximately 30 years ago was examined in our outpatient department of breast surgery for routine examination of the breasts. Ten years ago, she underwent BCS and axillary lymph node dissection of the right breast and axillae for breast cancer at a different institution. At that time, the operator aware that the patient was injected a foreign body for augmentation in her both breasts approximately 20 years ago, and the breasts had not color change, pain or hardness. Nevertheless, surgery was performed to treat the breast cancer, and after adjuvant chemotherapy, radio-oncologist proceeded with radiotherapy, while being aware of the patient's history before surgery. The final stage was IIA (pT2N0M0), and the subtype was luminal $\mathrm{A}$. The patient received four cycles of adjuvant chemotherapy (anthracyclinecyclophosphamide) and underwent radiation therapy (fraction dose, $180 \mathrm{cGy}$; total dose, 5,940 cGy; boost, 900 cGy) of the right breast for 7 weeks. She also received five-year adjuvant tamoxifen therapy. The patient had no complications until completion of therapy and she visited our department for breast screening. At that time, she had no abnormal magnetic resonance imaging (MRI) findings, as no recurrent breast cancer was observed with foreign body injection (Figure 1A). However, four years ago, she started noticing skin thickening in her right breast and bluish discoloration of the surrounding skin. After 2 years, she visited the outpatient department with increased skin discoloration. We considered about scleroderma, morphea and recurrent breast cancer for differential diagnosis. Biopsy was recommended; however, the patient refused.
Therefore, we decided to perform follow-up and imaging work-up. Follow-up breast MRI revealed no malignancy or recurrence (Figure 1B). Based on the imaging findings, we decided to conduct follow-up for 1 year. After 1 year, there was a skin lesion wider than that observed 2 years ago. Although the imaging study was benign (Figure 1C), we decided to operate on the right breast because of her concern for recurrent breast cancer and complaints of pain. We were aware that she had been injected with a foreign body in both breasts before surgery by asking the patient. She underwent a simple mastectomy of the right breast but refused to undergo reconstruction (Figure 1D). The patient had a successful postoperative course and was discharged without complications, and she was very satisfied with the progress of the surgery.

\section{Histologic findings}

The specimen size was $15.0 \mathrm{~cm} \times 11.0 \mathrm{~cm} \times 5.0 \mathrm{~cm}$, with skin (Figure 2A). No recurrent tumors were observed. There were heterogeneous cystic spaces with foreign materials, fat necrosis (Figure 2B). The overlying skin tissue had mild epidermal atrophy, sclerosis, and hyalinization of dermal collagen (Figure 2C). In the deep subcutaneous tissue, diffuse and wide range of fat necrosis with foreign body reactions (Figure 2D). For the differential diagnosis of amyloidosis, additional immunohistochemistry (Congo red) was performed, and the result was negative.

\section{Ethical statement}

All procedures performed in studies involving human participants were in accordance with the ethical standards of the institutional and/or national research committee(s) and with the Helsinki Declaration (as revised in 2013). Written informed consent was obtained from the patient for publication of this case report and accompanying images. A copy of the written consent is available for review by the editorial office of this journal.

\section{Discussion}

After BCS for breast cancer, radiotherapy may yield many adverse effects, including radiation-induced dermatitis, sclerodermatous changes, skin telangiectasia, atrophy, skin necrosis, and secondary malignancies $(2,4)$.

Scleroderma is a heterogeneous group of autoimmune fibrosing disorders, and its cause is unknown. This group of 

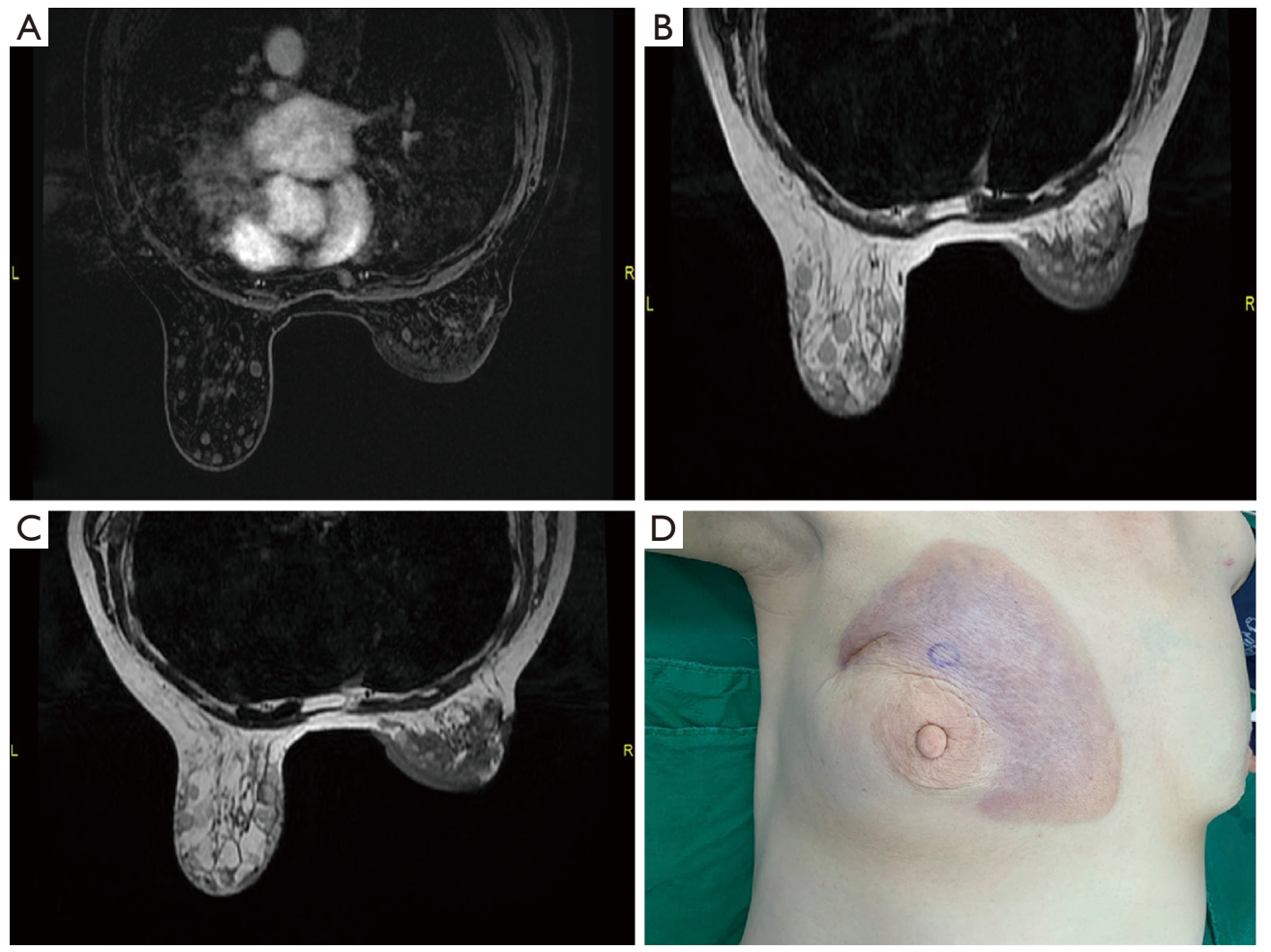

Figure 1 Magnetic resonance imaging and gross findings of the patient's breast. (A) In 2016, there was no abnormal enhancement in both breasts; (B) in 2019, there were multiple variable-sized $(0.2-1.5 \mathrm{~cm})$ injection granulomas in both breasts but no abnormal enhancement; (C) in 2021: postoperative status, right 12:00-1:00 and innumerable foreign body granulomas, both breasts; (D) in the operating room, the right breast shows hyperpigmentation with a bluish color.

disorders includes localized scleroderma, systemic sclerosis with limited cutaneous systemic sclerosis, and diffuse cutaneous systemic sclerosis subtypes (7). In scleroderma, autoimmune antibodies, including anti-nuclear antibodies (ANAs), are positive in serology, involve various organs, and are divided into systemic scleroderma and localized scleroderma (morphea) (7).

Systemic scleroderma is characterized by finger arthritis, and the dermis and epidermis are replaced by very thick sclerotic collagen; many inflammatory reactions also occur (8). The earliest and most frequent sign of systemic scleroderma is skin involvement, which is the "skin thickening of the fingers of both hands extending proximal to the metacarpophalangeal joints" criterion alone; it is considered sufficient for the diagnosis of systemic sclerosis, scoring 9/9 (9). Other cutaneous signs include fingernail alterations (80\%), cutaneous ulcerations (40\%), telangiectasia (75\%), hyperpigmentation of thickened skin (30\%), and cutaneous calcifications affecting soft tissues (25\%) (10). Approximately $95 \%$ of patients with systemic scleroderma presented with ANAs (positive finding: >1:160). In patients with diffuse cutaneous systemic scleroderma, extractable nuclear antigens and anti-Scl-70 antibodies are generally associated (11).

Localized scleroderma, also of unknown etiology, is a rare chronic connective tissue and autoimmune disease (12). It is limited to the skin and directly underlying tissues, such as subcutaneous tissue and bone (13). Morphea is a localized subtype of scleroderma, characterized by the deposition of thickened collagen and abnormal fibroblast activity (5). Plaque morphea or morphea "en plaque" is the most common variant of morphea in adults and is limited to the epidermis and dermis. Breast cancer is commonly observed in women. Early inflammatory lesions are well defined, and inflammation diminishes over time in the lesion center. The lesion continues as the stage subsides, and the resulting lesions are white, skinny sclerotic plaques with postinflammatory hyperpigmentation (14). The macroscopic findings of the skin lesion are similar to those in our case; however, there was a difference in that there was a white 

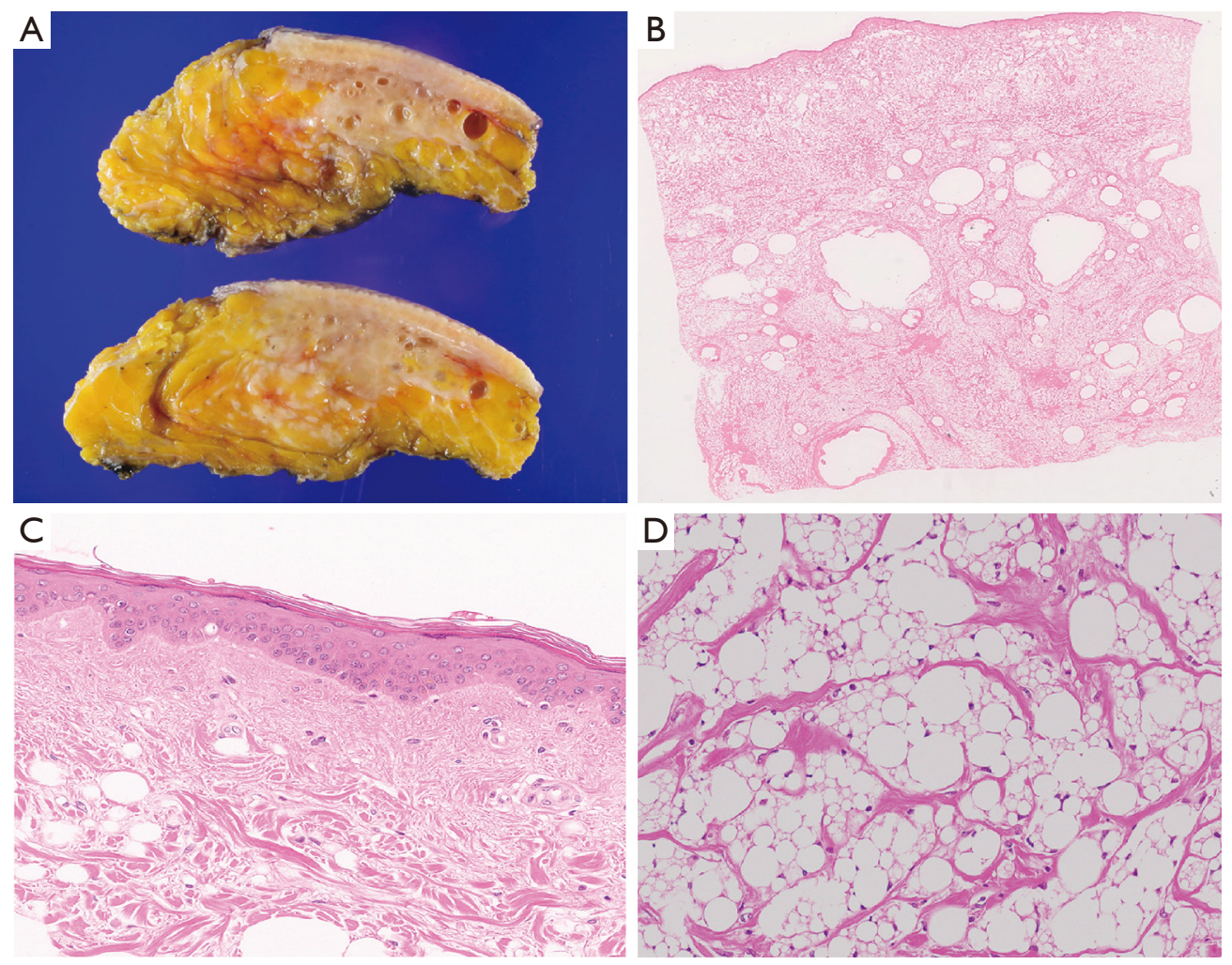

Figure 2 Gross finding and histopathologic findings of specimen after mastectomy. (A) On gross examination, the cut surface shows variable-sized cystic spaces containing oily material. The cystic spaces are surrounded by breast parenchyma with sclerotic and myxoid change; (B) the superficial dermis to the deep subcutaneous tissue is replaced by the heterogeneous cystic spaces with foreign materials $(\times 10$, hematoxylin and eosin); (C) on higher magnification, the overlying skin tissue shows mild epidermal atrophy, sclerosis, hyalinization of dermal collagen, and loss of follicular units. The possibility of chronic radiation dermatitis is suggested ( $\times 200$, hematoxylin and eosin); (D) on higher magnification of the deep subcutaneous tissue, there is diffuse and wide fat necrosis with the release of foreign materials into the intercellular spaces resulting in a foreign body reaction $(\times 200$, hematoxylin and eosin).

sclerotic scar-like lesion in the middle area.

Although morphea induced by radiation was first described in 1905, it was not recognized until 1989 as a complication of radiotherapy $(15,16)$. The incidence of PIM is approximately 1 in every 500 patients in a study that included 3,000 irradiated patients with breast cancer (17). The total radiation dose, age, and acute reaction to radiotherapy were not found as risk factors, whereas large breast, superficial tumor location, and higher fat content of the irradiated tissue seemed to be significant risk factors for developing PIM (5). Most patients developed PIM after BCS (18), and the same surgery was performed in our case, which had a lower fat content. The early stage presents clinically as painful erythematous plaques and histologically shows thickened eosinophilic collagen bundles in the reticular dermis (19). The last stage presents as skin induration with violaceous discoloration, skin retraction, and pigmentation of the breast (19). There are some theories for the development of PIM, including radiationinduced neo-antigen formation; this results in a pathogenic secretion of TGF- $\beta$, leading to fibroblast activation, collagen synthesis, and subsequent fibrosis (20). Because breast cancer recurrence is the most important differential diagnosis, a skin biopsy including a $4.0-\mathrm{mm}$ punch biopsy or incisional biopsy is required and typically cannot be ruled out radiologically $(21,22)$. However, in our case, the patient refused skin biopsy, and we only evaluated the radiologic and breast MRI findings. The histologic characteristics of PIM are as follows: (I) presence of thick collagen bundles in the reticular dermis and loss of adnexal structures and peri-adnexal adipose tissue; and (II) perivascular and periadnexal lymphoplasmacytic infiltrate (early phase) $(23,24)$. 
The treatment of PIM is variable, including corticosteroids, topical imiquimod, imatinib, colchicine, D-penicillamine, immunosuppressants, plasmapheresis, ultraviolet light A (UVA) 1, psoralen UVA therapy, and surgery (5). However, in our case, the patient did not want any other treatment and wanted surgery owing to growing discoloration and persistent pain. Therefore, we thought that the patient's lesion was a PIM and performed the surgery; however, the histologic result did not indicate PIM after surgery.

The differential diagnostic considerations include chronic radiodermatitis (CRD), radiation-induced fibrosis (RIF), post-irradiation pseudosclerodermatous panniculitis (PIPP), recurrent breast cancer, atypical vascular lesion (AVL), and angiosarcoma. CRD occurs weeks to years after radiation and has poikilodermatous features with epidermal involvement, distinct areas of epidermal atrophy, hyperplasia, and sclerosis of the dermis $(23,25)$. RIF occurs months to years after radiation and has erythema, edema, and induration findings with fibrosis of the deep subcutaneous tissue and fascia (24,25). PIPP usually occurs within a year after radiation and has an erythematous indurated plaque (26). Recurrent breast cancer occurs months to years after radiation and has erythema and induration features. AVL occurs 3 to 6 years after radiation and has erythematous to violaceous macules or papule clinical findings (27). Angiosarcoma occurs 4 to 8 years after radiation and has erythematous to violaceous nodules or plaque features (28).

Scleroderma-like disorders may arise owing to various etiologies. The pathologic findings are similar to those of scleroderma, and ANAs are negative (7). However, in our case, it was sclerotic to deep dermal fibrosis and subcutaneous tissue. Fat necrosis and foreign body reactions were observed from the epidermis to the superficial dermis. Furthermore, rather than sclerotic collagen, there were infiltration of fat and accompanying changes in collagen.

Subcutaneous oleoma is a foreign body reaction induced by liquid foreign materials, including vegetable oils, such as cotton seeds and sesame oils (6). Lesions are generally atypical and bizarre in appearance, and involvement of the breast is highly suspicious for factitious disease (29). On gross appearance, the hyperpigmentation in our case appeared similar to that described above (Figure 1D). The lesion's skin, dermis, and subcutaneous fat are usually involved, and the lesions are seen as isolated or coalescent hard, brown nodules, forming typical plaques. Thereafter, fat necrosis and suppuration occur, and the inflammatory process may cause granulomatous inflammation and fibrosis (6). Oleomas may have a painless mass or painful, hard swelling with skin ulceration. Patients may have asymptomatic lesions for 2-25 years. In our case, the patient's symptoms started approximately 30 years after the injection. Oleomas show cystic lesions on ultrasound as hypoechogenic lesions with acoustic shadowing accompanied by round hyperechoic encapsulated lesions with calcifications (30). Breast MRI findings after oil injection can be useful for revealing an artificial lesion. In a previous study, MRI showed that the main component of paraffinoma on both T1-weighted (T1-W) and T2weighted $(\mathrm{T} 2-\mathrm{W})$ images and the round component were hypointense on the T1-W and T2-W images with a remarkable suppression of fat saturation sequences (31). In our case, all the lesions showed injection granulomas in both breasts in the T2-W images (Figure 1B); however, it was difficult to determine whether it was a paraffinoma or an oleoma. Microscopically, there is granulomatous inflammation with multiple clear vacuoles and foreign body multinucleated giant cells (32). In our case, we do not know what material was injected into the patient's breasts. However, the pathologic findings were very similar to those of oleoma: (I) gross findings: cystic and oily findings and (II) tissue findings: fat necrosis in the subcutaneous tissue, release of fat droplets into intercellular spaces, and a granulomatous response. Moreover, it seems more reasonable to consider the discoloration as the skin thickens as a result of a foreign body reaction invading the superficial dermis and subcutaneous tissue rather than a lesion on the skin caused by autoimmune diseases, such as scleroderma.

The patient had a history of receiving radiotherapy after BCS 10 years ago, and it would be reasonable to diagnose her condition as oleoma with post-radiation fibrosis among drug-induced and toxic scleroderma-like disorders.

In conclusion, it can be difficult to distinguish between PIM and oleoma in patients with a complex history, as in our case. In addition, the diagnosis of oleoma may be challenging. In this case, the patient had both a history of radiotherapy and a history of foreign body injection, making the clinical diagnosis difficult. Finally, the final diagnosis was oleoma with post-radiation fibrosis among druginduced and toxic scleroderma-like disorders, which were the result of a combined effect of what happened 10 and 30 years ago, respectively. PIM and oleoma are nonmalignant but can impair a patient's quality of life owing to symptoms, such as skin thickening, pain, and distortion of the breast. Moreover, the clinical presentation is similar to that of local recurrence of breast cancer. Thus, imaging 
follow-up, skin punch biopsy, or surgery is needed for a definitive diagnosis.

\section{Acknowledgments}

Funding: None.

\section{Footnote}

Reporting Checklist: The authors have completed the CARE reporting checklist. Available at https://dx.doi. org/10.21037/gs-21-549

Peer Review File: Available at https://dx.doi.org/10.21037/ gs-21-549

Conflicts of Interest: All authors have completed the ICMJE uniform disclosure form (available at https://dx.doi. org/10.21037/gs-21-549). The authors have no conflicts of interest to declare.

Ethical Statement: The authors are accountable for all aspects of the work in ensuring that questions related to the accuracy or integrity of any part of the work are appropriately investigated and resolved. All procedures performed in studies involving human participants were in accordance with the ethical standards of the institutional and/or national research committee(s) and with the Helsinki Declaration (as revised in 2013). Written informed consent was obtained from the patient for publication of this case report and accompanying images. A copy of the written consent is available for review by the editorial office of this journal.

Open Access Statement: This is an Open Access article distributed in accordance with the Creative Commons Attribution-NonCommercial-NoDerivs 4.0 International License (CC BY-NC-ND 4.0), which permits the noncommercial replication and distribution of the article with the strict proviso that no changes or edits are made and the original work is properly cited (including links to both the formal publication through the relevant DOI and the license). See: https://creativecommons.org/licenses/by-nc-nd/4.0/.

\section{References}

1. Early Breast Cancer Trialists' Collaborative Group (EBCTCG), Darby S, McGale P, et al. Effect of radiotherapy after breast-conserving surgery on 10year recurrence and 15-year breast cancer death: metaanalysis of individual patient data for 10,801 women in 17 randomised trials. Lancet 2011;378:1707-16.

2. Harper JL, Franklin LE, Jenrette JM, et al. Skin toxicity during breast irradiation: pathophysiology and management. South Med J 2004;97:989-93.

3. Porock D, Kristjanson L. Skin reactions during radiotherapy for breast cancer: the use and impact of topical agents and dressings. Eur J Cancer Care (Engl) 1999;8:143-53.

4. Fehlauer F, Tribius S, Höller U, et al. Long-term radiation sequelae after breast-conserving therapy in women with early-stage breast cancer: an observational study using the LENT-SOMA scoring system. Int J Radiat Oncol Biol Phys 2003;55:651-8.

5. Spalek M, Jonska-Gmyrek J, Gałecki J. Radiation-induced morphea - a literature review. J Eur Acad Dermatol Venereol 2015;29:197-202.

6. Sarıca Ö, Kayhan A, Demirkürek HC, et al. Subcutaneous oleomas following sunflower oil injection: a novel case and review of literature. J Breast Health 2016;12:141-4.

7. Ferreli C, Gasparini G, Parodi A, et al. Cutaneous Manifestations of Scleroderma and Scleroderma-Like Disorders: a Comprehensive Review. Clin Rev Allergy Immunol 2017;53:306-36.

8. Rongioletti F, Gambini C, Micalizzi C, et al. Mucin deposits in morphea and systemic scleroderma. Dermatology 1994;189:157-8.

9. van den Hoogen F, Khanna D, Fransen J, et al. 2013 classification criteria for systemic sclerosis: an American College of Rheumatology/European League against Rheumatism collaborative initiative. Arthritis Rheum 2013;65:2737-47.

10. Desbois AC, Cacoub P. Systemic sclerosis: An update in 2016. Autoimmun Rev 2016;15:417-26.

11. Ho KT, Reveille JD. The clinical relevance of autoantibodies in scleroderma. Arthritis Res Ther 2003;5:80-93.

12. Careta MF, Romiti R. Localized scleroderma: clinical spectrum and therapeutic update. An Bras Dermatol 2015;90:62-73.

13. Fett N, Werth VP. Update on morphea: part I. Epidemiology, clinical presentation, and pathogenesis. J Am Acad Dermatol 2011;64:217-28; quiz 229-30.

14. Leitenberger JJ, Cayce RL, Haley RW, et al. Distinct autoimmune syndromes in morphea: a review of 245 adult and pediatric cases. Arch Dermatol 2009;145:545-50. 
15. Alhathlool A, Hein R, Andres C, et al. Post-Irradiation Morphea: Case report and review of the literature. $\mathrm{J}$ Dermatol Case Rep 2012;6:73-7.

16. Kushi J, Csuka ME. Generalized morphea after breast cancer radiation therapy. Case Rep Rheumatol 2011;2011:951948.

17. Davis DA, Cohen PR, McNeese MD, et al. Localized scleroderma in breast cancer patients treated with supervoltage external beam radiation: radiation port scleroderma. J Am Acad Dermatol 1996;35:923-7.

18. Mosterd K, Winnepenninckx V, Vermeulen A, et al. Morphea following surgery and radiotherapy: an evolving problem. J Eur Acad Dermatol Venereol 2009;23:1099-101.

19. Gonzalez-Ericsson PI, Estrada MV, Al-Rohil R, et al. Post-irradiation morphoea of the breast: a case report and review of the literature. Histopathology 2018;72:342-50.

20. Schaffer JV, Carroll C, Dvoretsky I, et al. Postirradiation morphea of the breast presentation of two cases and review of the literature. Dermatology 2000;200:67-71.

21. Laetsch B, Hofer T, Lombriser N, et al. Irradiationinduced morphea: $\mathrm{x}$-rays as triggers of autoimmunity. Dermatology 2011;223:9-12.

22. Walker D, Susa JS, Currimbhoy S, et al. Histopathological changes in morphea and their clinical correlates: Results from the Morphea in Adults and Children Cohort V. J Am Acad Dermatol 2017;76:1124-30.

23. Bleasel NR, Stapleton KM, Commens C, et al. Radiation-

Cite this article as: Lee HS, Jung EJ, Kim JM, Kim JY, Kim IK, Kim JR, Kim TH, Jang JY, Woo JW, Lee J, Park T, Jeong SH, Cho E, An HJ. Is it really post-irradiation morphea or oleoma of the breast?-A case report and literature review. Gland Surg 2021;10(12):3424-3430. doi: 10.21037/gs-21-549 induced localized scleroderma in breast cancer patients. Australas J Dermatol 1999;40:99-102.

24. Reddy SM, Pui JC, Gold LI, et al. Postirradiation morphea and subcutaneous polyarteritis nodosa: case report and literature review. Semin Arthritis Rheum 2005;34:728-34.

25. Walsh N, Rheaume D, Barnes P, et al. Postirradiation morphea: an underrecognized complication of treatment for breast cancer. Hum Pathol 2008;39:1680-8.

26. Shirsat HS, Walsh NM, McDonald LJ, et al. Postirradiation pseudosclerodermatous panniculitis with involvement of breast parenchyma: a dramatic example of a rare entity and a pitfall in diagnosis. J Cutan Pathol 2016;43:444-50.

27. Mandrell J, Mehta S, McClure S. Atypical vascular lesion of the breast. J Am Acad Dermatol 2010;63:337-40.

28. Seinen JM, Styring E, Verstappen V, et al. Radiationassociated angiosarcoma after breast cancer: high recurrence rate and poor survival despite surgical treatment with R0 resection. Ann Surg Oncol 2012;19:2700-6.

29. Benson EA. Artefactual breast disease. Br J Surg 1986;73:163.

30. Georgieva J, Assaf C, Steinhoff M, et al. Bodybuilder oleoma. Br J Dermatol 2003;149:1289-90.

31. Khong PL, Ho LW, Chan JH, et al. MR imaging of breast paraffinomas. AJR Am J Roentgenol 1999;173:929-32.

32. Requena L, Requena C, Christensen L, et al. Adverse reactions to injectable soft tissue fillers. J Am Acad Dermatol 2011;64:1-34; quiz 35-6. 